\title{
Análise de Sistemas de Informação aplicados à Gestão Portuária
}

Dissertação apresentada à Escola

Politécnica da Universidade de

São Paulo para obtenção do

Título de Mestre em Engenharia

São Paulo

2003 


\section{Análise de Sistemas de Informação aplicados à Gestão Portuária}

Dissertação apresentada à Escola

Politécnica da Universidade de

São Paulo para obtenção do

Título de Mestre em Engenharia

Área de Concentração:

Engenharia Naval e

Sistemas Oceânicos

Orientador:

Prof. Dr. Cláudio Mueller Prado Sampaio

São Paulo

2003 


\section{FICHA CATALOGRÁFICA}

Kurosawa, Rosane Santana da Silva

Análise de Sistemas de Informação Aplicados à Gestão Portuária

Município de São Paulo, São Paulo, 2003. $168 p$.

Dissertação (Mestrado) - Escola Politécnica da Universidade de São Paulo. Departamento de Engenharia Naval e Sistemas Oceânicos.

2.Sistema de Informação, 2.Gestão Portuária I. Universidade de São Paulo. Escola Politécnica. Departamento de Engenharia Naval e Sistemas Oceânicos II.t. 
Ao meu amado esposo Carlos, e as luzes de minha vida, meus filhos Shuichi e Masao pois juntos me deram todo amor, incentivo, carinho, compreensão e ajuda sem os quais nada eu realizaria. 


\section{AGRADECIMENTOS}

A Deus por tantas dificuldades vencidas.

Ao Prof. Dr. Cláudio Mueller Prado Sampaio, orientador e sábio, pelas diretrizes, permanente incentivo e apoio impar para que este trabalho se concretizasse.

Ao Prof. Dr. Orlando Fontes Lima Júnior pelo estímulo e colaboração. 


\section{RESUMO}

O presente trabalho analisa e compara casos de implantação de sistemas de informação em portos com perfis diferenciados, com o intuito de levantar os fatores relevantes para o sucesso da implantação da tecnologia da informação dentro do Sistema Portuário Mundial. O embasamento teórico, além de apresentar a fundamentação necessária para as análises, objetiva também, expor as diferenças importantes que têm marcado a sociedade. A análise do histórico das implantações e do atual estágio dos sistemas nos portos estudados, através de diversas entrevistas com os usuários, demonstrou fatores positivos que devem ser maximizados em qualquer implantação de tecnologia da informação. Demonstrou, também, fatores negativos que devem ser evitados para garantir o sucesso da implantação. A observância destes fatores levantados certamente irão garantir a conclusão bem sucedida de projetos que

provocarão mudanças organizacionais através da implantação de sistemas de informação em sistemas portuários. 


\begin{abstract}
This study analyzes and compares cases of information system implementations in port whose profiles are quite distinct, in order to raise the key factors for a successful IT implementation in the World Port Community. The theoretical background not only presents the foundation needed for the analyses, but also aims to expose the important differences that have characterized the society. The analysis of the record of implementations and of the present stage of the systems in the ports surveyed, through several interviews with users, has shown positive factors which must be maximized in any IT implementation. It has also shown negative factors that must be avoided in order to ensure a successful implementation. Compliance with the key factors is bound to guarantee a successful conclusion of projects which will bring about organizational changes through the implementation of IT systems in Port Community.
\end{abstract}




\section{LISTA DE TABELAS}

CAPÍTULO 2

PÁGINA

Tabela 2.1- Entrevistas Realizadas

\section{CAPÍTULO 3}

Tabela 3.1 - Evolução dos Sistemas de Informação e do Conceito de Informação 19

Tabela 3.2 - Redes Conceituais $\quad 21$

Tabela 3.3 - Funções e Objetivos das Camadas de Sistema de Comunicação 26

Tabela 3.4 - Domínio de Conectividade 26

Tabela 3.5 - Protocolos de Comunicação de Dados 28

Tabela 3.6 - Internet / $2^{\mathrm{a}}$. NGN/ $3^{\mathrm{a}}$. NGN 30

Tabela 3.7 - Diferenças entre "Internet" vs. "Intranet" vs. "Extranet" 31

\section{CAPÍTULO 4}

Tabela 4.1 - Organizações envolvidas no Sistema Portuário Mundial (Porto de Rotterdan)

Tabela 4.2 - Os 10 Maiores Portos Mundiais em Movimentação de Contêineres

Tabela 4.3 - Movimentação de Carga Processada por Homem/hora (Principais Portos)

Tabela 4.3 - Implantação de Sistemas de Informação no Sistema Portuário Mundial

Tabela 4.4 - Descrição dos Subsistemas Funcionais integrantes do Sistema KL-Net

Tabela 4.5 - Sistemas Eletrônicos de Informação Implantados no Sistema Portuário Mundial

Tabela 4.6 - Sistemas de Informação Implantados no Sistema Portuário Brasileiro 


\section{LISTA DE FIGURAS}

CAPÍTULO 1

PÁGINA

Figura 1.1 - Estrutura do Trabalho

06

\section{CAPÍTULO 2}

Figura 2.1 - Estrutura Seqüencial do Projeto de Pesquisa

\section{CAPÍTULO 3}

$\begin{array}{ll}\text { Figura } 3.1 \text { - Modelo cliente/servidor } & 20\end{array}$

Figura 3.2 - Arquitetura de Rede LAN e Rede WAN por cabeamento 22

Figura 3.3 - Arquitetura de Rede LAN e Rede WAN combinação sem fio e cabeamento

Figura 3.4 - Arquitetura de Rede LAN e Rede WAN totalmente sem fio 23

Figura 3.5 - Arquitetura de Rede "Token-Ring" 24

Figura 3.6 - Arquitetura de Rede "Ethernet" 24

Figura 3.7 - Exemplo de Uso de Protocolo de Comunicação e Dados 27

Figura 3.8. - VoIP - Integração do Universo da Voz e dos dados 31

Figura 3.9 - (a) Simbologia Composta / (b) Simbologia RSS 33

Figura 3.10 - Cartões Inteligentes "Smart Card" 34

Figura 3.11 - Etiquetas Inteligentes "Smart Tag" 34

Figura 3.12 - Aplicações da Biometria Digital 35

Figura 3.13 - Aplicações da Biometria Digital em Sistemas de Informação 36

Figura 3.14 - Concepção da troca eletrônica de informações (EDI) 37

Figura 3.15 - Linguagem UN/EDIFACT: United Nations Electronic Data 38

Figura 3.16 - Integração de EDI e XML 41

Figura 3.17 - EDI Tradicional 42

Figura 3.18 - EDI -Estação de Trabalho 44

Figura 3.19 - Web EDI 45

Figura 3.20 - EDI através da Internet 46

$\begin{array}{ll}\text { Figura 3.21 - Combinação EDI/XML } & 47\end{array}$ 


\section{CAPÍTULO 4}

Figura 4.1 - Ciclo de Exportação e Importação

Figura 4.2 - Processo de Exportação / Importação no Sistema Portuário Mundial 52

Figura 4.3 - Configuração do Sistema de Informação "DAKOSY" 68

Figura 4.4 - Arquitetura do Sistema de Informação "DAKOSY" 70

Figura 4.5 - Arquitetura do Sistema de Informação "ZAPP” 71

Figura 4.6 - Configuração do Sistema de Informação “ADEMAR” 73

Figura 4.7 - Arquitetura do Sistema de Informação "INTIS" 76

Figura 4.8 - Estrutura do Processo Customs Container Scan 77

Figura 4.9 - Estrutura do Sistema "SEAGHA" 79

Figura 4.10 - Imagem Digital do Porto de Antuérpia 80

Figura 4.11 - Conceito do Virtual Port Community - VPC 81

Figura 4.12 - Arquitetura do Sistema de Informação "SEAGHA" 82

Figura 4.13 - Arquitetura do Sistema de Informação "SEA" 85

Figura 4.14 - Ilustração do Microchip - Smart Tag 86

Figura 4.15 - Estrutura do Sistema de Informação "TRADENET" 88

Figura 4.16 - Visual Gate Truck $\quad 89$

Figura 4.17 - Estrutura do Sistema de Informação "BHT" 92

Figura 4.18 - Configuração do Sistema de Informação "KL-Net” " 94

Figura 4.19 - Conceito do sistema Port-MIS 96

Figura 4.20 - Conceito do sistema EIPOS 96

Figura 4.21 - Conceito do sistema KL-Net e subsistema ATOMOS 97

Figura 4.22 - Estrutura do Sistema de Informação "PortIC" 99

Figura 4.23 - Sistema de Informação “ MarNet” no Porto de Lisboa 101

Figura 4.24 - Fluxo da Informação e Processo Documental de Carga / EUA 103

Figura 4.25 - Estrutura da Supervia Eletrônica de Dados - SED 107

Figura 4.26 - Estrutura do Sistema de Informação "Carga On-Line" 109

Figura 4.27 - Estrutura do Sistema de Informação "COSMOS” 111 


\section{CAPÍTULO 5}

$\begin{array}{ll}\text { Figura 5.1 - Etapas do Processo Decisório } & 116\end{array}$

Figura 5.2 - Aplicação de Sistemas de Informação nas Organizações 118

Figura 5.3 - Estrutura para Procedimentos de Embarque - União Européia 121

Figura 5.4 - Estrutura do Sistema SEAGHA para Procedimentos do Manifesto 122

Figura 5.5 - Estrutura do Sistema DAKOSY para Procedimentos do Manifesto 124

Figura 5.6 - Comunidade Virtual e Sistema Financeiro / Porto de Rotterdam 125

Figura 5.7 - Estrutura do Sistema DAKOSY para Procedimentos do Manifesto 126

Figura 5.8 - Estrutura do Sistema SEA para Procedimentos do Manifesto 127

Figura 5.9 - Estrutura do Sistema BHT para Procedimentos do Manifesto 128

Figura 5.10 - Arquitetura do Fluxo do Manifesto Eletrônico - SED 129 


\section{SUMÁRIO}

RESUMO

ABSTRACT

LISTA DE TABELAS

LISTA DE FIGURAS

CAPÍTULO 1 - INTRODUÇÃO $\quad 01$ a 07

$\begin{array}{ll}1.1 \text { Considerações Iniciais } & 01\end{array}$

$\begin{array}{ll}1.2 \text { Tema e Problema } & 02\end{array}$

$\begin{array}{ll}1.3 \text { Objetivos da Pesquisa } & 03\end{array}$

1.3.1 Objetivo Geral 03

$\begin{array}{ll}\text { 1.3.2 Objetivos Específicos } & 03\end{array}$

$\begin{array}{ll}1.4 \text { Limitações da Pesquisa } & 04\end{array}$

$\begin{array}{ll}1.5 \text { Justificativa e Importância } & 04\end{array}$

$\begin{array}{ll}1.6 \text { Estrutura da Dissertação } & 05\end{array}$

CAPÍTULO 2 - METODOLOGIA DE PESQUISA $\quad 08$ a 15

2.1 - Metodologia da Pesquisa 08

$\begin{array}{ll}2.2 \text { - Projeto de Pesquisa } & 09\end{array}$

2.3 - Procedimento de Coleta de Dados 13

CAPÍTULO 3 - TECNOLOGIAS DE INFORMAÇÃO 16 a 48

3.1 - Tecnologia da Informação: Definições e Conceitos 16

3.2 - Rede de Computadores 19

3.3 - INTERNET/INTRANET 29

3.4 - Sistemas Inteligentes de Armazenamento e Aquisição de Informações 32

3.4.1 - Código de Barras 32

3.4.2 - Cartões Inteligentes ("Smart Cards") e

Etiquetas Inteligentes ("Smart Tags") 33

3.4.3 - Biometria Digital 35

3.5 - Transferência Eletrônica de Dados ("Eletronic Data Interchange - EDI") 36 
3.5.2 - Linguagem XML - Extensible Markeup Language 39

3.5.3 - Comparações e Aplicações dos Sistemas Linguagens EDI e XML 40 3.6 - Sistemas Eletrônicos de Troca de Informação - SETI 41

3.6.1 - Sistema Eletrônico de Troca de Informação - EDI Integrado 42

3.6.2 - Sistema Eletrônico de Troca de Informação - Estação de Trabalho 43

3.6.3 - Sistema Eletrônico de Troca de Informação através da Internet (Web EDI) 44

3.6.4 - EDI através da Internet 45

3.6.5 - Combinação XML / EDI 46

$\begin{array}{ll}3.7 \text { - Conclusões } & 47\end{array}$

CAPÍTULO 4 - O SISTEMA PORTUÁRIO MUNDIAL $\quad 49$ a 115

4.1 Introdução 49

4.2 Atividades Básicas do Sistema Portuário $\quad 50$

4.3 Integrantes do Sistema Portuário 53

4.4 O Sistema Portuário Mundial 56

4.5 Cenário do Sistema Portuário Brasileiro $\quad 57$

4.6 Análise da Competitividade do Sistema Portuário Mundial 58

4.6.1 Análise do Sistema Portuário Brasileiro e o Sistema Portuário Mundial 61

4.7 Modernização dos Portos através da Implantação de Sistemas Eletrônicos

de Troca de Informação $\quad 64$

4.7.1 Porto de Hamburgo - Alemanha 65

4.7.1.1 O Sistema Eletrônico de Informação "DAKOSY" 67

4.7.2 Porto de Lê Havre - França

4.7.2.1 O Sistema Eletrônico de Informação “ADEMAR” 72

4.7.3 Porto de Rotterdam - Holanda 74

4.7.3.1 O Sistema Eletrônico de Informação "INTIS"

4.7.4 Porto de Antuérpia-Bélgica 78

4.7.4.1 O Sistema Eletrônico de Informação "SEAGHA" 79

4.7.5 Porto de Yokohama - Japão 83

4.7.5.1 O Sistema Eletrônico de Informação "SEA"

$\begin{array}{ll}\text { 4.7.6 Porto de Cingapura - Cingapura } & 87\end{array}$ 
4.7.6.1 O Sistema Eletrônico de Informação "TRADENET"

4.7.7 Porto de Bremen - Alemanha 90

4.7.7.1 O Sistema Eletrônico de Informação "BHT” 91

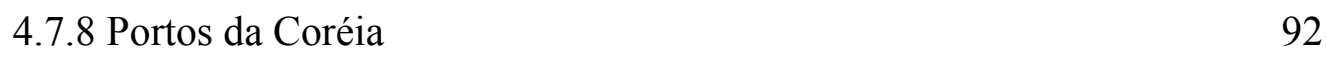

4.7.8.1 O Sistema Eletrônico de Informação “KL-Net” 93

4.7.9 Porto de Barcelona - Espanha 98

4.7.9.1 O Sistema Eletrônico de Informação "PortlC" 98

$\begin{array}{ll}\text { 4.7.10 Porto de Lisboa -Portugal } & 100\end{array}$

4.7.10.1 O Sistema Eletrônico de Informação "MarNet" 100

4.7.11 O Sistema Portuário dos Estados Unidos da América 102

4.7.11.1 O Sistema Eletrônico de Informação "DTEDI” 103

4.8 Modernização dos Portos Brasileiros através da Implantação de Sistemas Eletrônicos Troca de Informação 104

4.8.1 Porto de Santos - Estado de São Paulo 104

4.8.1.1 Supervia Eletrônica de Dados - SED 105

4.8.2 Porto de Paranaguá - Estado do Paraná 108

4.8.2.1 Sistema de Informação "Carga On-Line” 108

4.8.3 Porto de Sepetiba - Estado do Rio de Janeiro 110

4.8.3.1 Sistema de Informação "COSMOS" 110

$\begin{array}{ll}4.9 \text { Conclusões } & 113\end{array}$

CAPÍTULO 5 - SISTEMAS DE INFORMAÇÃO E A GESTÃO PORTUÁRIA 115 a 131

5.1 Importância dos Sistemas de Informação nas Organizações 115

5.2 Sistemas de Informação Aplicados à Gestão Portuária 120

5.2.1 Porto de Antuérpia - Manifesto via Sistema SEAGHA 122

5.2.2 Porto de Hamburgo - Manifesto via SISTEMA DAKOSY 123

5.2.3 Porto de Rotterdam - Manifesto via SISTEMA INTIS 125

5.2.4 Porto de Yokohama - Manifesto via SISTEMA SEA 126

5.2.5 Porto de Bremen - Manifesto via SISTEMA BHT 127

5.3 Procedimentos do Manifesto via SED no Porto de Santos 128

5.4 Dificuldades e Deficiências dos Sistemas Portuários Aplicados à

$\begin{array}{ll}\text { Gestão Portuária } & 130\end{array}$

$\begin{array}{ll}5.5 \text { Conclusões } & 131\end{array}$ 
CAPÍTULO 6 - CONSIDERAÇÕES FINAIS

132 a 133

LISTA DE REFERÊNCIAS

133 a 136

APÊNDICE A

137 a 138

GLOSSÁRIO

139 a 143 